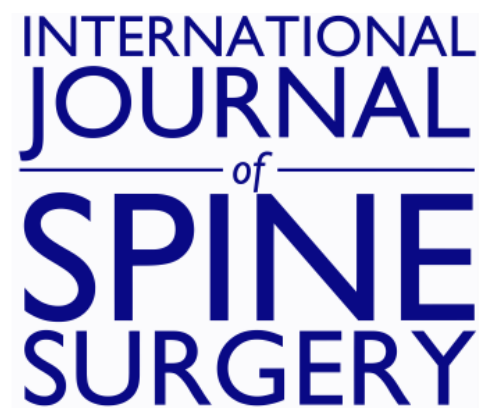

\title{
Osteochondroma Arising from the Thoracic Transverse Process
}

Ding Wenyuan, Li Baojun, Shen Yong, Zhang Wei and Zhang Yingze

Int J Spine Surg 2009, 3 (1) 12-16

doi: https://doi.org/10.1016/SASJ-2008-0026-CR

http://ijssurgery.com/content/3/1/12

This information is current as of April 26, 2023.

Email Alerts Receive free email-alerts when new articles cite this article. Sign up at:

http://ijssurgery.com/alerts

The International Journal of Spine Surgery

2397 Waterbury Circle, Suite 1,

Aurora, IL 60504, Phone: +1-630-375-1432

(C) 2009 ISASS. All Righfso Refservededom http://ijssurgery.com/ by guest on April 26, 2023 


\title{
Osteochondroma Arising from the Thoracic Transverse Process
}

Ding Wenyuan, MD, ${ }^{a}$ Li Baojun, MD, ${ }^{a}$ Shen Yong, PhD, MD, ${ }^{a}$ Zhang Wei, PhD, MD, and Zhang Yingze, MD ${ }^{b}$

\begin{abstract}
The incidence of osteochondroma is rare and only $2 \%$ of such tumors are found in the spine area. When they are found in the vertebral column, less than $1 \%$ of all osteochondromas and few tumors occur in the thoracic vertebrae. An osteochondroma arising from the transverse process of the vertebra is even rarer, especially following from the thoracic transverse process. Here we report a giant solitary osteochondroma arising from the thoracic transverse process of T8 vertebra and involving the corresponding transverse process and rib.
\end{abstract}

A 28-year-old man presented with a progressive thoracic node, and neuroradiological evaluation of the spine showed a giant mass lesion involving the transverse process of T8 vertebra and concomitant corresponding facet joint and rib on the left side. At surgery, a firm and cartilaginous tumor originating from the transverse process was radically excised and surgical curettage of the lesion was performed.

It is concluded that accurate and prompt diagnosis requires a high index of suspicion followed by surgical treatment to prevent severe morbidity in cases of primary spinal column tumors. The histological examination of this patient revealed the lesion was osteochondroma.

The best choice of treatment for spinal osteochondromas is surgical excision or curettage and spinal stabilization, if necessary.

Key Words: Osteochondroma; Spine tumor; Process; Appendix of vertebral. SAS Journal. March 2009;3:11-15. DOI: SASJ-2008-0026-CR

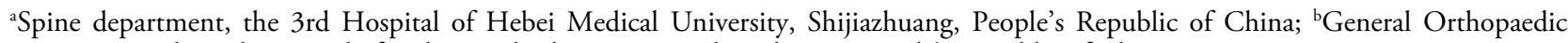
Department, the 3rd Hospital of Hebei Medical University, Shijiazhuang, People's Republic of China.

Address correspondence and reprint requests to Yingze Zhang, MD, General Orthopaedic Department, the 3rd Hospital of Hebei Medical University, 139 Ziqiang Road, Shijiazhuang, People’s Republic of China. E-mail: baojunli@gmail.com

No funds were received in support of this work. No benefits in any form have been or will be received from a commercial party related directly or indirectly to the subject of this manuscript.

\section{INTRODUCTION}

Although osteochondromas are the most common benign tumor of the bone and often occur in the long bone, only $2 \%$ of such tumors are found in the spinal area. ${ }^{1}$ When they do occur in the vertebral column, less than $1 \%$ of all osteochondromas and few tumors present in the thoracic vertebrae. $^{2}$ These lesions, solitary or multiple, are often asymptomatic and have a predilection for the cervical region. ${ }^{3}$ However, an osteochondroma arising from the transverse process--specifically, the thoracic transverse process--is even rarer. ${ }^{4}$ In this report, we present a rare case of a giant thoracic solitary osteochondroma arising from the transverse process of T8 vertebra and involving the corresponding facet joint and rib on the left side, along with a complete review of the literature.

\section{CASE HISTORY}

We report on a 28 -year-old man suffering from intermittent back pain due to a node in the thoracic vertebrae. After a 1-month treatment of anti-inflammatory drugs, the symptoms had not subsided and the node had begun to increase in size and could be detected at the epidermis. Pain did not increase. On physical examination, there was a considerable tenderness over the lower thoracic spine, but there was no neurological deficit. The location of the node was $1 \mathrm{~cm}$ on the left side of the transverse process. Palpation results showed it was hard and about $1 \mathrm{~cm} \times 2 \mathrm{~cm}$ in size. The erythrocyte sedimentation rate (ESR) was $13 \mathrm{~mm} / \mathrm{h}$ (normal, $<15 \mathrm{~mm} / \mathrm{h}$ ), and the white blood cell (WBC) count was normal. The results of liver function tests and serum chemistry including blood glucose and urinalysis were normal. An agglutination test for Salmonella and Brucella yielded negative results.

A plain X-ray showed what appeared to be an uneven ovoid paraspinal mass at the level of T8 and T9 vertebrae (Fig. 1). Computed tomography (CT) of the thoracic spine, performed to further characterize the lesion, revealed high attenuation, arising from the left lateral aspect of the T8 transverse process overlying the left eighth rib posteriorly, with central soft-tissue density and a calcified rim. This suggested a primary bone tumor with a calcificated part evection into the thoracic cage (Fig. 2). A $3 \mathrm{D}$ reconstruction of the $\mathrm{CT}$ results also confirmed it was an osteochondroma coming from the transverse process (Fig. 3). Magnetic resonance imaging (MRI) of the 


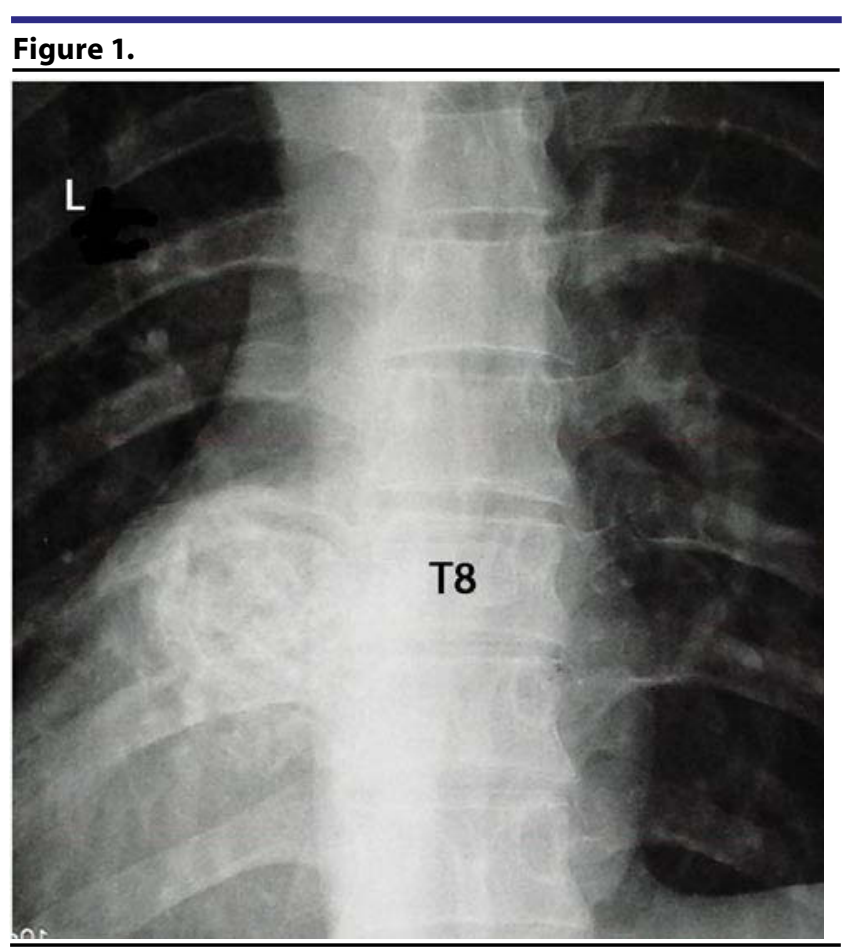

The X-ray of this patient shows there is a round, high-density background in the left part of the T8 vertebra.

spine showed a giant mass lesion involving the transverse process of $\mathrm{T} 8$ and concomitant corresponding vertebral body and rib on the left side. The lesion was isointense with the adjacent vertebral body marrow on T2-weighted images and slightly hyperintense to normal marrow on T1weighted images. A thin hypointense rim surrounding the lesion on T1-weighted images represented a thin shell of cortical bone surrounding the lesion. A mild improvement of the lesion is seen on the post-contrast sagittal T1-

\section{Figure 2.}

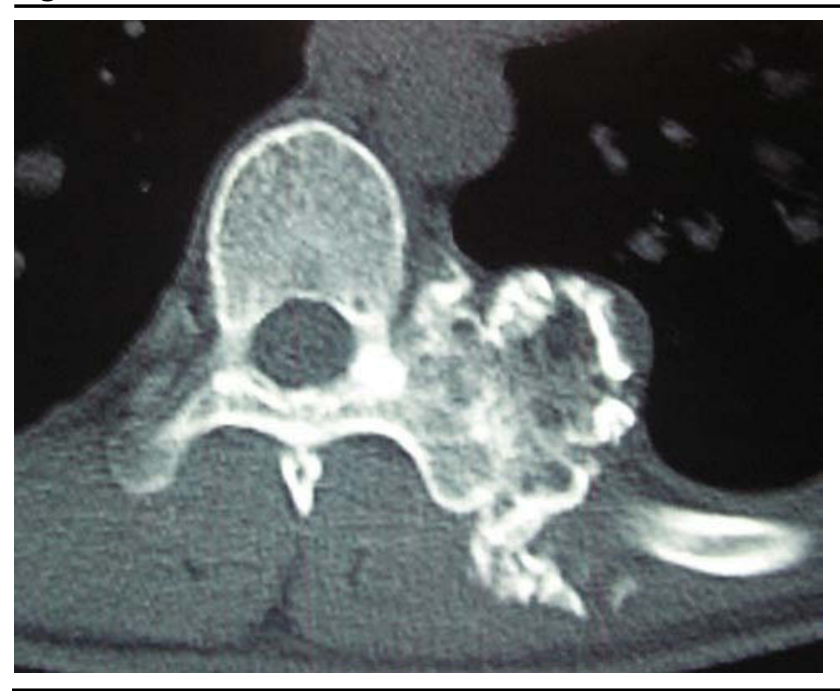

The CT scan suggests the outline of the T8 left transvertebral is irregular. In some local area lateral to the internal thoracic cave, the basement becomes wider and its density is uneven, so we can see something of the high bone density. The T8-T9 left intervertebral foramen becomes narrow.

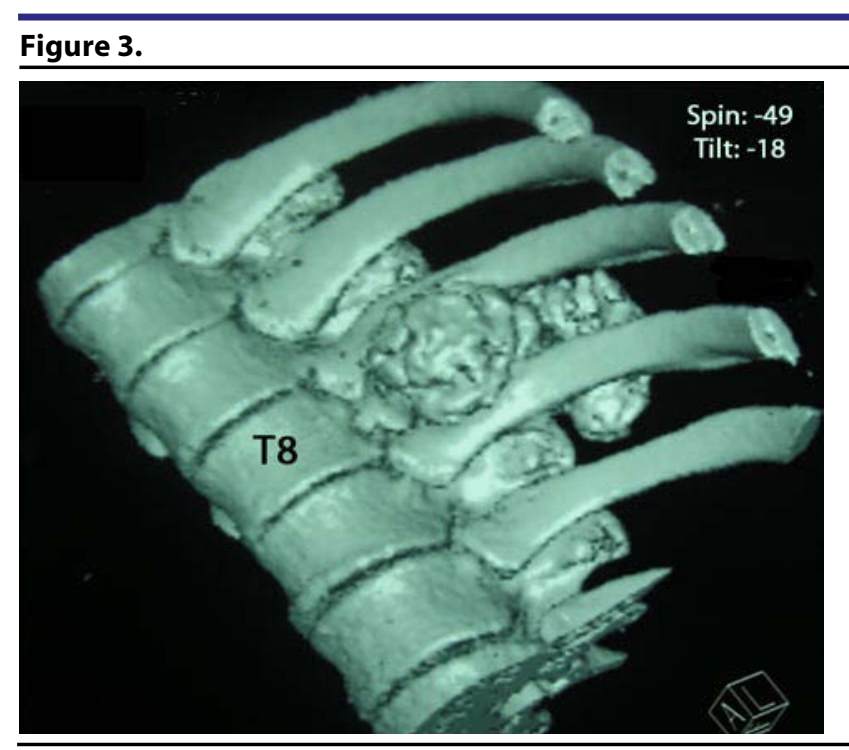

The 3D CT reconstruction of this patient shows the osteochondroma of T8 vertebra comes from the transverse process.

weighted images (Fig. 4). The aspiration-needle biopsy results showed the existence of cartilage cells.

During the surgery, the back part of the lesion resembled a pearly brown marble. It was adherent to the lateral aspect of the vertebral body and adjacent to pleura and aorta. It shelled out easily; however, the former part

\section{Figure 4.}

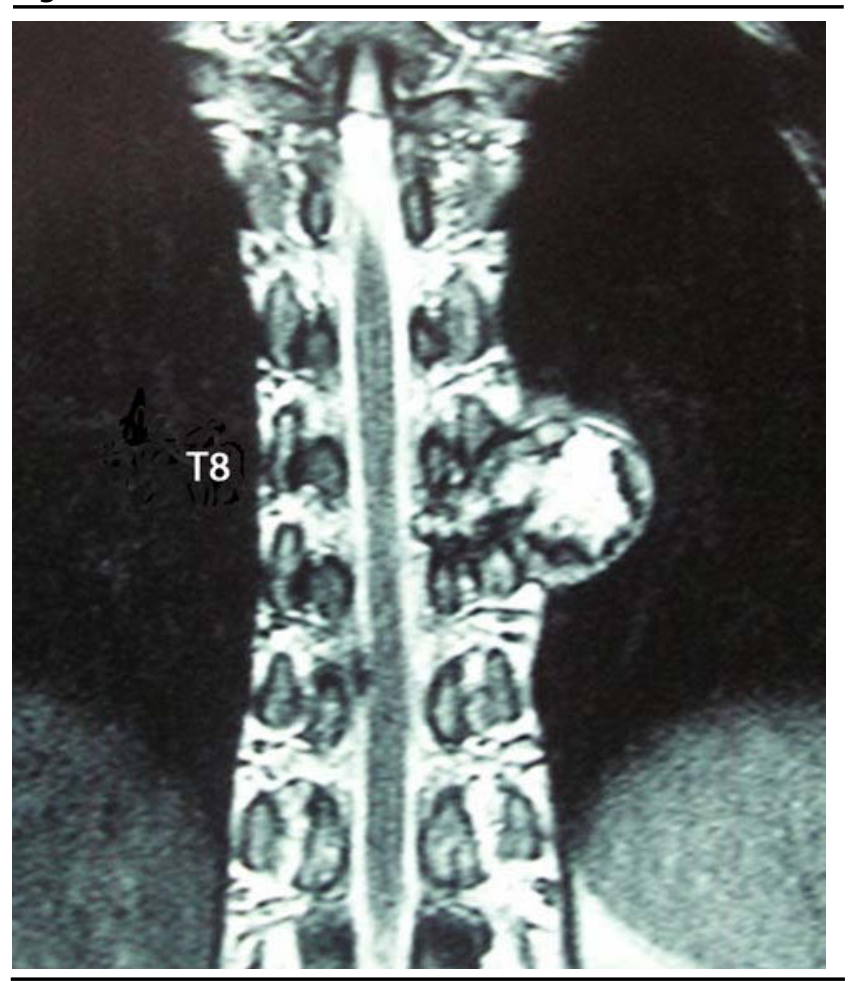

This patient's MRI results suggest the left T8 vertebral transverse patterns widened. The local area of the disease is irregularly prone to the thorax, which was within its uneven $\mathrm{T} 1, \mathrm{~T} 2$ mixed abnormal signal to the dorsal protruding lesions, which showed high signal intensity on T1 or T2. T8-9 left intervertebral foramen was narrow and compressed. 
within the thoracic cage was more difficult to remove (Fig. 5). The patient underwent a backside incision with about $\mathrm{cm}$ of the eighth and ninth ribs excised from the back. The osteocartilaginous tumor originating from the transverse process was radically excised and surgical curettage of the lesion performed. The adherence between the osteochondroma cap and the membrana pleuralis was broken during separation and should heal naturally. The lesion was resectioned in two parts, about $3 \mathrm{~cm} \times 3 \mathrm{~cm} \times 2.5$ $\mathrm{cm}$ in total (Fig. 6). Pathological specimens revealed the lesion to be an osteochondroma with a thin cartilaginous cap (Fig. 7). The patient recovered fully without residual deficit. Follow-up plain X-ray and CT of the thoracic spine showed complete removal of the tumor.

\section{Figure 5.}

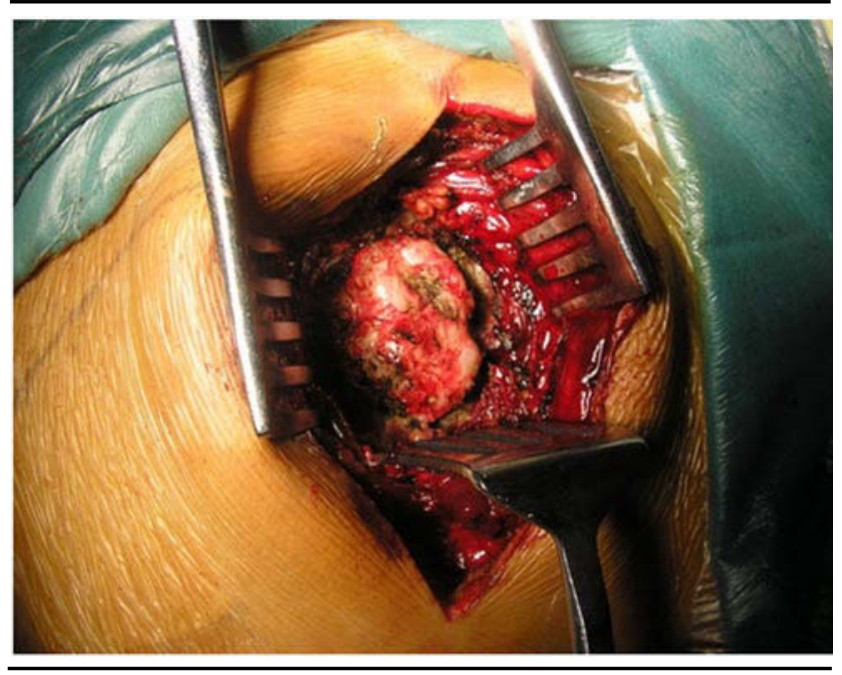

It seems the metamorphic hard surface and the cartilage cap exist in this lesion.

\section{DISCUSSION}

Osteochondromas arise because of a developmental defect in the growth plate. This defect is characterized by an osteocartilaginous growth that originates near the end of a long bone and grows away from the joint. ${ }^{5}$ Histologically, these lesions have central lamellar bone and peripheral cartilage with evidence of enchondral ossification of the bone and cartilage. ${ }^{6}$ The most common site of origin of spinal osteochondromas is the neural arch, and the transverse process is a rare site of origin. Our case is atypical, as the giant lesion arose from the left transverse process of the thoracic vertebra. To the best of our knowledge, no cases of spinal osteochondroma growing from the transverse process have been reported previously in the literature.

Clinically, the first manifestation of spinal osteochondromas is back pain over the involved area of the spine, but in this case, the pain of the affected area was not important. Leong and Thng et al. ${ }^{7}$ reported a case of cervical osteochondroma manifesting as neck pain with no neurological deficit. In a review of the English

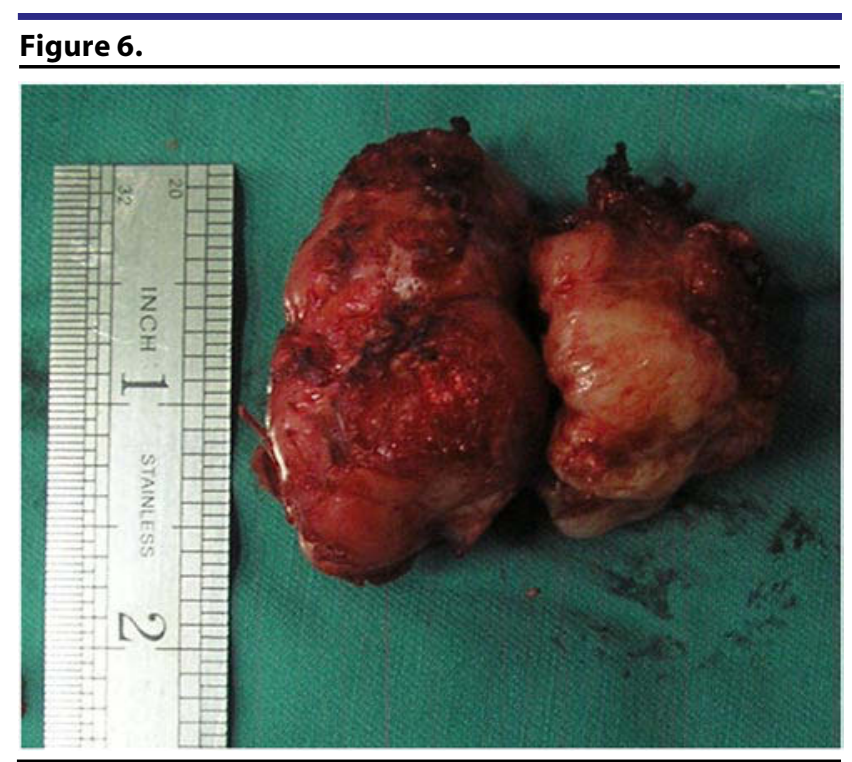

The pathological tissues taken out in the operation.

Figure 7.

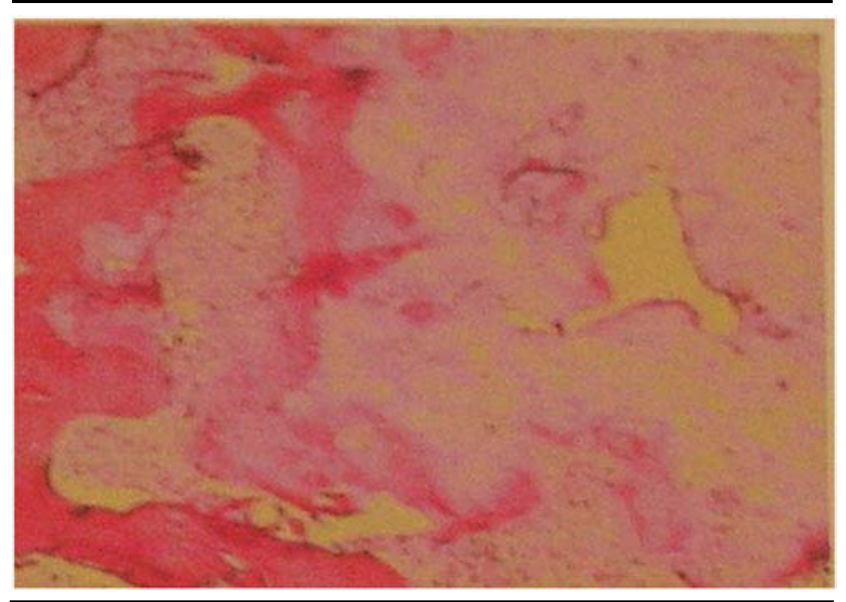

The postoperative pathological result confirms the diagnosis of osteochondroma.

language medical literature, Khosla et al. found 41 published cases of separate vertebral osteochondromas presenting with spinal cord compression. ${ }^{8}$ Clinical signs of spinal cord compression consisting of weakness, numbness and sphincter dysfunction could be seen in some cases.

Fiechtl et al. reported a case of an 8-year-old girl with hereditary multiple exostoses presenting with only atypical spinal curvature. ${ }^{9}$ Therefore, the diagnosis of spinal osteochondroma cannot be made on clinical grounds alone because of the wide range of etiologies and the difficulty in localizing the lesion.

Radiologically, plain radiographs and CT are useful in describing the extent of affected bone and in preoperative planning. CT reveals a well-circumscribed lobulated mass with areas of chondral mineralization and calcification, 
but it does not provide a detailed look at cartilage. A benign cartilaginous cap is usually only several millimeters thick and not detectable on CT scans, but it may be thicker. In larger lesions with bulky cartilaginous caps as well as in lesions that recur after resection, a benign degeneration should be suspected. MRI is useful in cases with spinal osteochondromas and a cartilaginous cap that appears hypointense on T1-weighted and hyperintense on T2-weighted imaging. The two strongest clues to the diagnosis of spinal osteochondroma are first, the contiguous marrow signal intensity extending from the vertebral body into the lesion seen on T1weighted and T2-weighted images, and second, the dense mineralization of the lesion on CT scans. Nevertheless, a correct preoperative diagnosis remains difficult in those with spinal osteochondromas, as seen in our case.

The cornerstone of treatment of spinal osteochondromas is total excision or surgical curettage of the lesion. Both have shown low recurrence rates. In the presented case, surgical intervention was effective in resolving the patient's symptoms. Surgical decompression of the spinal cord and surgical stabilization of the spine are treatment choices in cases of spinal osteochondromas with clinical signs of spinal cord compression.

In conclusion, this case displays the usefulness of CT and MRI for the diagnosis of thoracic spinal osteochondromas arising from the transverse process. As in our case, early diagnosis and therapy would most likely lessen the chance of permanent neurologic shortage from a spinal osteochondroma.

The best choice of treatment for spinal osteochondromas is surgical excision or curettage and spinal stabilization, if necessary.

This manuscript was submitted October 13, 2008, and accepted for publication February 9, 2009.

\section{REFERENCES}

1. Milde T, Alamo L, Stadelmann C, Schweigerer L, Witt O. Multifocal osteochondroma after repeated irradiation in a boy with Hodgkin's disease. J Pediatr Hematol Oncol. 2005;27:344-345.

2. Ahmed AR, Tan TS, Unni KK, Collins MS, Wenger DE, Sim FH. Secondary chondrosarcoma in osteochondroma: report of 107 patients. Clin Orthop Relat Res. 2003;411:193-206.

3. Moon KS, Lee JK, Kim YS, et al. Osteochondroma of the cervical spine extending multiple segments with cord compression. Pediatr Neurosurg. 2006;42:304-307.

4. Brastianos P, Pradilla G, McCarthy E, Gokaslan Z. Solitary thoracic osteochondroma: case report and review of the literature. Neurosurgery. 2005;56:E1379.

5. Glick R, Khaldi L, Ptaszynski K, Steiner GC. Dysplasia epiphysealis hemimelica (Trevor disease): a rare developmental disorder of bone mimicking osteochondroma of long bones. Hum Pathol. 2007;38:1265-1272.
6. Agrawal A, Dwivedi SP, Joshi R, Gangane N. Osteochondroma of the sacrum with a correlative radiographic and histological evaluation. Pediatr Neurosurg. 2005;41:46-48.

7. Leong L, Thng P. A deceptive cervical lymph node: a solitary spinal osteochondroma. Ann Acad Med Singapore. 2002;31:611-613.

8. Khosla A, Martin DS, Awwad EE. The solitary intraspinal vertebral osteochondroma. An unusual cause of compressive myelopathy: features and literature review. Spine. 1999;24:77-81.

9. Fiechtl JF, Masonis JL, Frick SL. Spinal osteochondroma presenting as atypical spinal curvature: a case report. Spine. 2003;28:E252E255. 


\section{EXTENDED REFERENCES}

Diagnostic imaging of solitary tumors of the spine: what to do and say.

Rodallec MH, Feydy A, Larousserie F, Anract P, Campagna R, Babinet A, Zins M, Drapé JL.

Metastatic disease, myeloma, and lymphoma are the most common malignant spinal tumors. Hemangioma is the most common benign tumor of the spine. Other primary osseous lesions of the spine are more unusual but may exhibit characteristic imaging features that can help the radiologist develop a differential diagnosis. Radiologic evaluation of a patient who presents with osseous vertebral lesions often includes radiography, computed tomography (CT), and magnetic resonance (MR) imaging. Because of the complex anatomy of the vertebrae, CT is more useful than conventional radiography for evaluating lesion location and analyzing bone destruction and condensation. The diagnosis of spinal tumors is based on patient age, topographic features of the tumor, and lesion pattern as seen at CT and MR imaging. A systematic approach is useful for recognizing tumors of the spine with characteristic features such as bone island, osteoid osteoma, osteochondroma, chondrosarcoma, vertebral angioma, and aneurysmal bone cyst. In the remaining cases, the differential diagnosis may include other primary spinal tumors, vertebral metastases and major nontumoral lesions simulating a vertebral tumor, Paget disease, spondylitis, echinococcal infection, and aseptic osteitis. In many cases, vertebral biopsy is warranted to guide treatment.

Spinal osteochondroma: spectrum of a rare disease.

Srikantha U, Bhagavatula ID, Satyanarayana S, Somanna $S$, Chandramouli $B A$.

Osteochondromas are the most common benign bone tumor. Although the metaphysial region of long bones is the usual site of these tumors, the vertebrae may be infrequently affected. The presentation may vary from typical compressive myelopathy to radiculopathy or radiculomyelopathy, depending on the site of involvement. The authors present 3 consecutive cases of cervical spine osteochondromas encountered over 3 years at their institution, each different in its site of involvement, presentation, and chosen treatment. The patient in Case 1 had the typical presentation and lesion site, and was treated with a conventional laminectomy. The patient in Case 2 presented with an extensive disease that required complex, staged surgery with spinal fusion and instrumentation. The patient in Case 3 presented with monoradiculopathy and had a facet joint osteochondroma that was successfully treated with a simple partial facetectomy, without laminectomy.

Osteochondroma of the sacrum: a case report and review of the literature.

Samartzis D, Marco RA.

STUDY DESIGN: A case report and review of the literature. OBJECTIVES: To describe the en bloc excision and postoperative outcome of an osteochondroma of the sacrum compressing the neural elements, as well as review the literature on solitary osteochondroma involving the sacrum. SUMMARY OF BACKGROUND DATA: Osteochondroma is the most common primary benign bone tumor. However, this tumor rarely involves the spine and even more rarely involves the sacrum. To the best of our knowledge, en bloc excision of a solitary osteochondroma of the sacrum has not been previously reported. METHODS: An 11-year-old male presented with disabling radicular pain in the right lower extremity. Radiologic studies showed a lesion occurring from the sacral lamina that was compressing the S2 nerve root. The tumor was excised en bloc through a posterior approach. The cavitary defect within the sacrum was reconstructed with crushed cancellous allograft and demineralized bone matrix putty. A literature review of solitary sacral osteochondroma was conducted of the Englishbased medical literature. RESULTS: Histologic studies showed the tumor to be an osteochondroma. After surgery, pain was completely relieved, and neurologic function was normal. At the last follow-up, the sacroiliac joint remained intact, and there was no evidence of local recurrence. A literature review revealed 4 previous cases addressing osteochondroma of the sacrum. CONCLUSIONS: Osteochondroma is a rare primary benign bone tumor that can occur in the sacrum. Local contamination and, therefore, the likelihood of local recurrence, are decreased when an en bloc, as opposed to an intralesional, excision is performed.

\section{Spinal osteochondromas in middle-aged to elderly patients.}

\section{Sakai D, Mochida J, Toh E, Nomura T.}

STUDY DESIGN: Three case reports of solitary spinal osteochondromas in middle-aged and elderly patients. OBJECTIVES: To describe the treatment of three rare cases of spinal osteochondromas in patients significantly older than the cases previously reported in the literature. SUMMARY OF BACKGROUND DATA: Osteochondromas arising in the vertebral column are considered rare. The mean age of clinical appearance is said to be in the second or third decade of life. METHODS: Clinical history, physical examinations, plain radiographs, magnetic resonance imaging, myelography, computed tomographic myelography, and histopathologic findings for each case were reviewed in conjunction with previous cases reported in the literature. RESULTS: Total removal of the tumor was effective in causing the symptoms to disappear. CONCLUSIONS: Spinal osteochondromas are rare and tend to appear in young adults. Three cases of spinal osteochondromas that were unusual in terms of age at clinical presentation and localization were reported, suggesting a continuous growth of the tumor beyond skeletal maturity. 